\title{
Left pneumonectomy and middle lobectomy for bronchiectasis
}

\author{
E. U. KÜRKLÜ and B. T. LE ROUX \\ The Thoracic Unit, Wentworth Hospital, Durban, and the Department of Surgery, \\ University of Natal
}

\begin{abstract}
Kürklü, E. U., and le Roux, B. T. (1973). Thorax, 28, 535-536. Left pneumonectomy and middle lobectomy for bronchiectasis. Left pneumonectomy and middle lobectomy were undertaken in a child for bilateral bronchiectasis of this extent. The thoracic surgeon who works among a large community medically ill served and among whom respiratory disease is common is frequently called upon to manage bronchiectasis surgically.
\end{abstract}

In the seven-year period $1966-72$, in the Thoracic Surgical Unit in Durban, Natal, 200 resections have been undertaken for bronchiectasis in a population numerically in excess of four million but only a proportion of whom are available for medical surveillance. Lobectomy was the commonest operation undertaken (100 patients); pneumonectomy was undertaken in 80 patients and segmental resection in 20. Bilateral resection was undertaken in 12 patients. In the same period

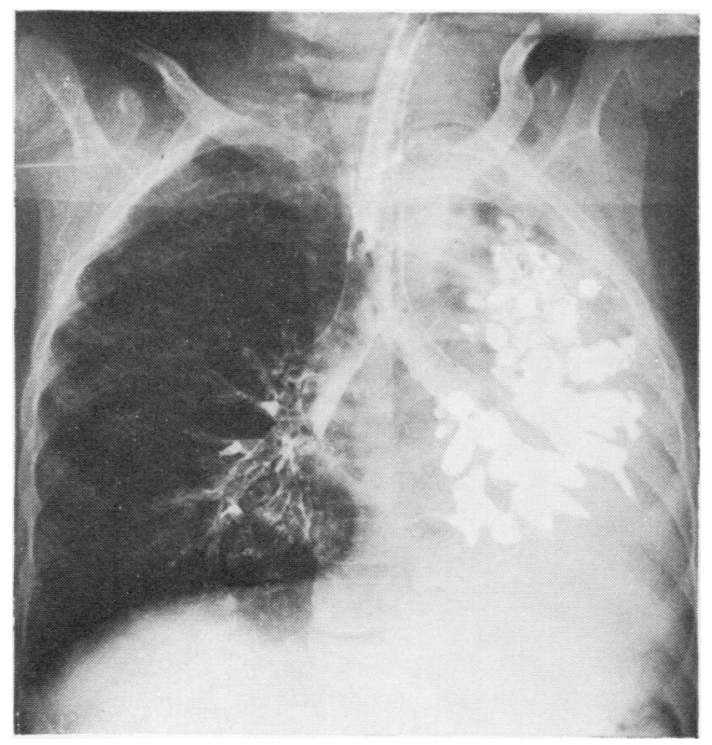

FIG. 1. Left oblique bronchogram showing total left bronchiectasis. in the same population group only 230 pulmonary resections were undertaken for bronchial carcinoma.

Experience of bronchiectasis in 1,038 patients managed in the Thoracic Surgical Unit at Edinburgh between 1947 and 1963 (le Roux, 1969)resection was undertaken in 409 of the patients -includes the report of one patient submitted to middle lobectomy and left pneumonectomy. Resection of this extent is unusual. The purpose of

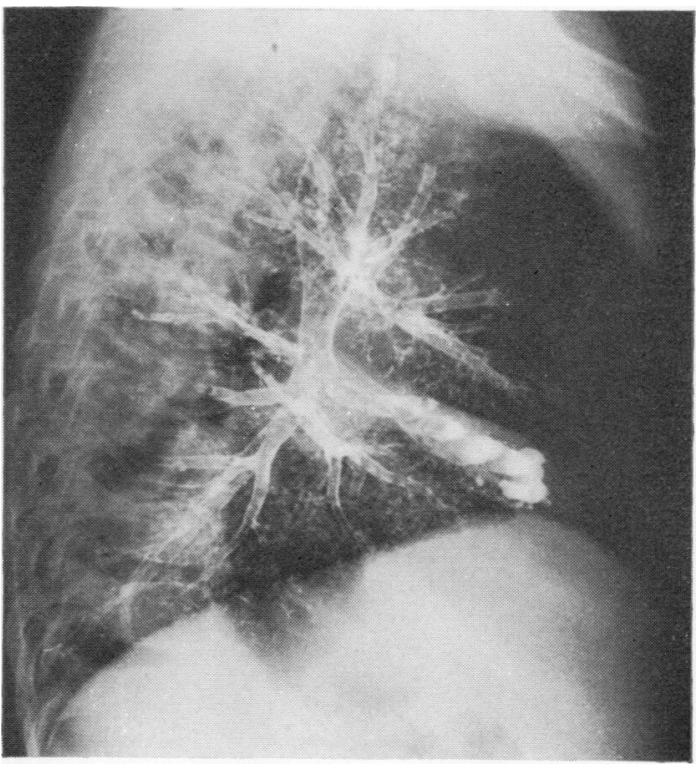

FIG. 2. Right lateral bronchogram showing middle lobar bronchiectasis. 
this paper is to report similar management of bilateral bronchiectasis.

\section{CASE REPORT}

A 9-year-old Bantu girl had had respiratory symptoms for much of her life and for the preceding year these had been ingravescent. Radiographically the mediastinum was deviated far to the left, the left lung was shrunken and opaque, and the middle lobe was also shrunken and opaque.

Bronchography demonstrated total left (Fig. 1) and middle lobar bronchiectasis (Fig. 2). It was elected to proceed to surgical management. Left pneumonectomy was undertaken after a period of preparation for operation. The operation was uneventful and recovery from this was rapid. Four weeks later middle lobectomy was undertaken. Recovery from this procedure was equally uneventful and a year later the child is in every respect well and symptom free. Histologically the left lung showed the appearance of fibrosis and bronchiectasis with foetalization of alveoli. The middle lobe was shrunken, with focal bronchiectasis and focal lymphocytic aggregates, fibrosis, collapse, emphysema, and destruction of lung tissue.

The anatomical limits of pulmonary resection are well established. It perhaps needs re-emphasis that in the management of bronchiectasis by resection the patients should have symptoms which can be attributed only to bronchiectasis, and surgical results are best when the disease is limited to shrunken lobes and segments devoid of respiratory function.

When faced with the need for pneumonectomy and contralateral lobectomy in the management of bronchiectasis, to remove first the grosser sump seems rational, since in a unilaterally totally bronchiectatic, shrunken lung there cannot be functional advantage, only suppurative embarrassment. Subsequent middle lobectomy, involving thoracotomy on the side which harbours the patient's only functioning lung, proved neither technically difficult nor, for the patient, uncomfortable. The postoperative radiographic appearances were indistinguishable from those preoperatively except for absence of the shadow of a shrunken airless middle lobe.

\section{REFERENCES}

le Roux, B. T. (1969). Bronchiectasis: the place of surgery in its management. Medical Proceedings, 15, 23. 